\title{
MÉTODO T-ODA NA MENSURAÇÃO DA EVIDENCIAÇÃO AMBIENTAL DAS EMPRESAS BRASILEIRAS LISTADAS NO IBRX-100 DA BM\&FBOVESPA - UM ESTUDO MULTICRITÉRIO
}

\author{
Mara Vogt \\ Universidade Regional de Blumenau - FURB, Blumenau - SC, Brasil \\ maravogtcco@gmail.com \\ Nelson Hein \\ Universidade Regional de Blumenau - FURB, Blumenau - SC, Brasil \\ hein@furb.br \\ Fabricia Silva da Rosa \\ Universidade Federal de Santa Catarina - UFSC, Florianópolis - SC, Brasil \\ fabriciasrosa@hotmail.com \\ Larissa Degenhart \\ Universidade Regional de Blumenau - FURB, Blumenau - SC, Brasil \\ lari_ipo@hotmail.com
}

\begin{abstract}
Resumo
O objetivo deste estudo foi de mensurar o grau de evidenciação ambiental das empresas brasileiras listadas no IBrX-100 da BM\&FBovespa utilizando o método de análise multicritério T-ODA. Trata-se de um estudo descritivo, documental e quantitativo. A amostra compreendeu as 97 empresas brasileiras pertencentes ao índice IBrX-100 listadas na BM\&FBovespa nos anos de 2010 a 2013. A partir dos resultados verificou-se que as empresas com maior grau de evidenciação ambiental em cada ano são: Ambev (2010), Duratex (2011), BRF Brasil Foods (2012) e a Eletrobrás (2013). Além disso, as organizações divulgaram diferentes informações nos períodos analisados, o que pode indicar seleção de informações visando a legitimação, pois as empresas tornam-se legitimadas quando demonstram que suas atividades estão de acordo com as demandas sociais. Além disso, as empresas poderão aumentar o seu grau de evidenciação se divulgarem mais informações em seus relatórios sobre suas ações ambientais, principalmente sobre o aspecto transporte.
\end{abstract}

Palavras-chave: $\quad$ Grau de Evidenciação Ambiental; Empresas Brasileiras; Relatório de Sustentabilidade; Relatório Anual.

\begin{abstract}
The objective of this study was to measure the degree of environmental disclosure of Brazilian companies listed on the IBrX-100 BM \& FBovespa using the multi-criteria analysis method T-ODA. This is a descriptive, document and quantitative study. The sample consisted of the 97 Brazilian companies belonging to the IBrX-100 index listed on the BM \& FBovespa in the years 2010 to 2013 . From the results it was found that the companies with the highest degree of environmental disclosure each year are: Ambev (2010), Duratex (2011), BRF Brazil Foods (2012) and Eletrobrás (2013). In addition, organizations reported different information in the analyzed periods, which may indicate selection of information aimed at legitimizing, as companies become legitimized when they demonstrate that their activities comply with social demands. In addition, companies can increase their degree of disclosure for disclosing more information in their reports on their environmental actions, especially on the aspect of transport.
\end{abstract}




\section{INTRODUÇÃO}

Nos últimos 30 anos o impacto das empresas sobre o meio ambiente tornou-se uma preocupação cada vez maior entre ambientalistas, legisladores, clientes, autoridades públicas e a sociedade em geral. Diante disso, há 15 anos as empresas começaram a evidenciar mais sobre as estratégias ambientais em função das pressões institucionais. Essas estratégias são relatadas por meio de indicadores e podem ser classificadas de acordo com o compromisso ambiental que representam (ALBERTINI, 2013).

Nossa (2002) ressalta que a preocupação com o meio ambiente e a conscientização encontra-se cada vez mais presente na sociedade. É por isso que as empresas investem em programas ambientais, pois descobrem que poluir é o mesmo que desperdiçar, não ter eficiência e não ter como competir. Afinal, a sociedade pressiona o Governo e as empresas, sobre a preservação do meio ambiente e essa pressão aumenta a partir do momento em que a poluição causada pelas atividades das organizações também for aumentar. É nesse momento que as empresas estabelecem metas para minimizar os impactos ambientais sobre as emissões de efluentes líquidos e gasosos, reciclagem de materiais, dentre outras, com vistas a legitimar suas atividades.

De acordo com Rosa et al. (2013), na década de 1990, o foco das informações ambientais apresentadas pelas empresas estava pautada na divulgação das políticas, dos objetivos, das responsabilidades profissionais e o cumprimento legal. Já na década de 2000, além dessas preocupações com a gestão e os aspectos legais, iniciaram as pesquisas relacionadas aos níveis de evidenciação, aos tipos de relatórios utilizados, os padrões de comunicação, os sistemas de gestão ambiental e as certificações. Nessa época, também foram pesquisados aspectos de desempenho ambiental, tais como os resíduos, a gestão de impactos e energia.

A partir de 2010, aumentaram consideravelmente os estudos sobre os critérios para avaliar o desempenho da empresa. Nesse período estudos passaram a analisaram a relação de causa e efeito das atividades das organizações com o meio ambiente (ROSA et al., 2013). Segundo Rosa et al. (2014), o interesse dos pesquisadores tem aumentado por aspectos relacionados ao desempenho ambiental como: água, energia, biodiversidade, emissões, efluentes, resíduos, impactos e transporte. No entanto, outros aspectos, como a certificação, os sistemas de gestão ambiental e as políticas ambientais, continuam fazendo parte dos grandes movimentos, debates sociais e políticos para o desenvolvimento sustentável.

Diante do contexto apresentado emerge a seguinte questão que norteia esta pesquisa: Qual é o grau de evidenciação ambiental das empresas brasileiras listadas no IBrX-100 da BM\&FBovespa? No intuito de responder a esta questão, o objetivo do estudo consiste em mensurar o grau de evidenciação ambiental das empresas brasileiras listadas no IBrX-100 da BM\&FBovespa.

O estudo justifica-se, pois as informações contidas nos relatórios deveriam ser além de amplas, exatas e verdadeiras, visto que muitos investidores utilizam as informações contidas nos relatórios ambientais para a tomada de decisões, decidindo se devem investir ou não em determinada empresa (NOSSA, 2002).

Justifica-se ainda devido ao fato do método de análise multicritério Trade-Off Decision Analysis (T-ODA), ser inédito dentro do tema evidenciação ambiental em pesquisas realizadas em âmbito nacional e internacional e devido a escassez de estudos na área contábil envolvendo o método. Conforme Rossoni (2011), o método é relevante, visto que a história nos mostra que os processos de tomada de decisão, geralmente não levam em consideração todos os critérios que modificariam certa decisão. Ressalta ainda que os gestores normalmente seguem a sua intuição para tomar decisões e, para que uma empresa tenha sucesso é preciso 
considerar esses critérios e ponderá-los corretamente.

\section{EVIDENCIAÇÃO AMBIENTAL}

Até o começo do século XX houve pouca preocupação das empresas quanto a preservação e recuperação do meio ambiente. Isso porque naquela época os recursos naturais eram considerados abundantes e os gestores utilizavam esses recursos como uma fonte inesgotável de matéria-prima a um custo muito baixo ou até mesmo nulo. A partir do momento que os efeitos da agressão ao meio ambiente começaram a se manifestar, a sociedade começou a cobrar das organizações maior responsabilidade ambiental. O mercado selecionou as empresas que estavam comprometidas com a preservação, manutenção e recuperação da natureza, visto que as demais comprometeriam o futuro do planeta (COSTA, 2006).

Essa crescente preocupação sobre os impactos da empresa na sociedade e no meio ambiente levou o público a exigir destas um comportamento social e ambientalmente responsável (GARCÍA-SÁNCHEZ; FRÍAS-ACEITUNO; RODRÍGUEZ-DOMÍNGUEZ, 2013).

A contabilidade pode ser vista como um importante instrumento para controlar e proporcionar as melhores decisões para as empresas sobre as suas atividades. As informações ambientais são de interesse dos fornecedores, Governo, bancos, investidores, sociedade, dentre outros, isso porque podem além de afetar o patrimônio das empresas, evidenciar os impactos ambientais causados e os incentivos das suas atividades em prol do meio ambiente (COSTA, 2006).

Dessa forma, para que as empresas se tornem competitivas, estas precisam evidenciar informações que sejam relevantes aos usuários para auxiliar na tomadas de decisões. Para as empresas apresentarem à sociedade seus compromissos, estas podem utilizar diversos recursos, como campanhas e divulgações em propagandas, divulgação de relatórios específicos, entre outros. Cada vez as organizações visam apresentar mais informações sobre suas atividades na buscar de se diferenciarem das demais, com relação às questões ambientais e sociais (KRESPI et al., 2012).

Nos últimos anos a evidenciação ambiental se expandiu em todo mundo de forma significativa (WANG; BERNELL, 2013). Mesmo que a evidenciação ambiental deveria ocorrer sempre, no Brasil, a legislação não obriga as empresas a divulgarem. Há uma tendência mundial na qual os investidores procuram as empresas que são socialmente responsáveis, sustentáveis e rentáveis para aplicarem os seus recursos (OLIVEIRA; MACHADO; BEUREN, 2012). Isso porque, de acordo com Meng et al. (2013), o nível de evidenciação das informações ambientais reflete o grau de responsabilidade ambiental corporativa das empresas.

A evidenciação voluntária das informações sociais e ambientais é um exemplo de regras e estruturas que são implementadas pelas empresas para responder as pressões sociais. Essas pressões, juntamente com a aceitação cada vez maior das estruturas de divulgação como o GRI, incentivam as empresas a divulgarem mais informações sobre a sustentabilidade (VILLIERS; LOW; SAMKIN, 2014).

Rosa et al. (2013) expõem que a evidenciação ambiental é um meio ou processo baseado em um conjunto de critérios que podem ser utilizados para avaliar a forma como as empresas influenciam e são influenciadas pelo ambiente. É uma forma de apresentar à sociedade como suas atividades são gerenciadas no que concerne o meio ambiente.

Da mesma forma, Rosa et al. (2014) frisam que a evidenciação pode ser conceituada como um meio, ou então um conjunto de meios utilizados por diferentes empresas, com o intuito de expor suas práticas ambientais aos stakeholders, ao mesmo tempo que funciona como ferramenta para a tomada de decisões aos diferentes interessados por essas informações. A evidenciação ambiental é baseada na economia, desenvolvimento sustentável e informação 
divulgada. Serve para os investidores realizarem previsões e analisarem o desempenho ambiental da empresa.

\section{PROCEDIMENTOS METODOLÓGICOS}

Diante do objetivo de mensurar o grau de evidenciação ambiental das empresas brasileiras listadas no IBrX-100 da BM\&FBovespa, realizou-se uma pesquisa descritiva, documental e com abordagem quantitativa.

\subsection{População E Amostra}

A população da pesquisa compreende todas as empresas pertencentes ao Índice Brasil 100 (IBrX-100) listadas na BM\&FBovespa, ou seja, 100 companhias. No entanto, pelo fato das empresas Bradesco, Klabin e Oi estarem duplicadas, a amostra do estudo foi composta por 97 destas empresas listadas na Bolsa de Valores de São Paulo, pertencentes ao índice IBrX-100. Por meio do Quadro 1 são apresentadas as empresas que compõem a amostra da pesquisa.

\begin{tabular}{|c|c|c|c|}
\hline \multicolumn{4}{|c|}{ Empresas } \\
\hline Aes Tietê & CPFL Energia & Itaú Unibanco Holding & PDG Realty \\
\hline All América Latina Log. & Cyrela Realty & JBS & Petróleo Brasileiro \\
\hline Ambev & Diagnósticos da América & Klabin & Petropar \\
\hline Anhanguera Educ. Part. & Duratex & Kroton Educacional & Porto Seguro \\
\hline Arteris & Ecorodovias & Light & Qualicorp \\
\hline B2W Companhia Digital & Eletrobrás Participações & LLX Log & Raia Drogasil \\
\hline Banrisul & Eletropaulo & Localiza Rent a Car & Randon \\
\hline BM\&FBovespa & Embraer & Lojas Americanas & Rossi Residencial \\
\hline BR Malls Participações & EDP - Energias do Brasil & Lojas Renner & Sabesp \\
\hline BR Properties & Eneva & M. Dias Branco & CIA Siderúrgica \\
\hline BCO Bradesco & Equatorial Energia & Magazine Luiza & Souza Cruz \\
\hline BCO Brasil & Estácio Participações & Marcopolo & Sul América \\
\hline BCO Santander & Even & Marfrig Global Foods & Suzano Papel e Celulose \\
\hline Bradespar & Eztec & Mills & Taesa \\
\hline Braskem & Fibria Celulose & Minerva & Telefônica Brasil \\
\hline BRF-Brasil Foods & Gafisa & MMX & TIM Participações \\
\hline Brookfield Incorporações & Gerdau & MRV & Totvs \\
\hline $\mathrm{CCR}$ & Gerdau Metalúrgica & Multiplan & Tractebel Energia \\
\hline Cemig & Gol & Multiplus & Ultrapar Participações \\
\hline Cetip & HRT Petróleo & Natura Cosméticos & Usiminas \\
\hline CIA Hering & Hypermarcas & Odontoprev & Vale \\
\hline Cielo & Iguatemi & OGX Petróleo e Gás & Valetron \\
\hline Copasa & Iochpe Maxion & $\mathrm{OI}$ & Valid \\
\hline Copel & Itausa Investimentos Itaú & Pão de Açúcar - CBD & Weg \\
\hline Cosan & & & \\
\hline
\end{tabular}

Fonte: Dados da pesquisa.

\subsection{Coleta E Análise dos Dados}

Verificou-se em todos os relatórios divulgados pelas empresas da amostra nos anos de 2010 a 2013, seja no Relatório Anual (RA) ou no Relatório de Sustentabilidade (RS), as informações apresentadas sobre as Emissões, Efluentes, Resíduos, Produtos/Serviços e Transportes. Os dados coletados foram inseridos em planilhas do software excel a partir da interpretação das diretrizes do GRI, na qual constam os aspectos, seus critérios e subcritérios, a descrição de cada um destes e os níveis de divulgação que foram verificados em cada relatório de cada empresa analisada.

Vale destacar que esses critérios e subcritérios apresentam diferentes escalas ordinais para expressar ordem entre os níveis, conforme o desempenho a ser medido em cada critério, o que possibilita a atribuição de até oito níveis distintos. Destaca-se que os níveis, escalas, foram criados a partir da interpretação de todas as informações apresentadas sobre cada aspecto analisado nas diretrizes do GRI (2013).

As escalas utilizadas variam entre 1 a no máximo 8 , dependendo do aspecto analisado, visto que alguns dos aspectos e seus critérios e subcritérios necessitam de escalas 
diferenciadas entre si, pois são informações distintas. Na Figura 1 são apresentados os aspectos e a devida mensuração e descrição dos critérios e subcritérios.

Figura 1 - Indicadores de Evidenciação Ambiental

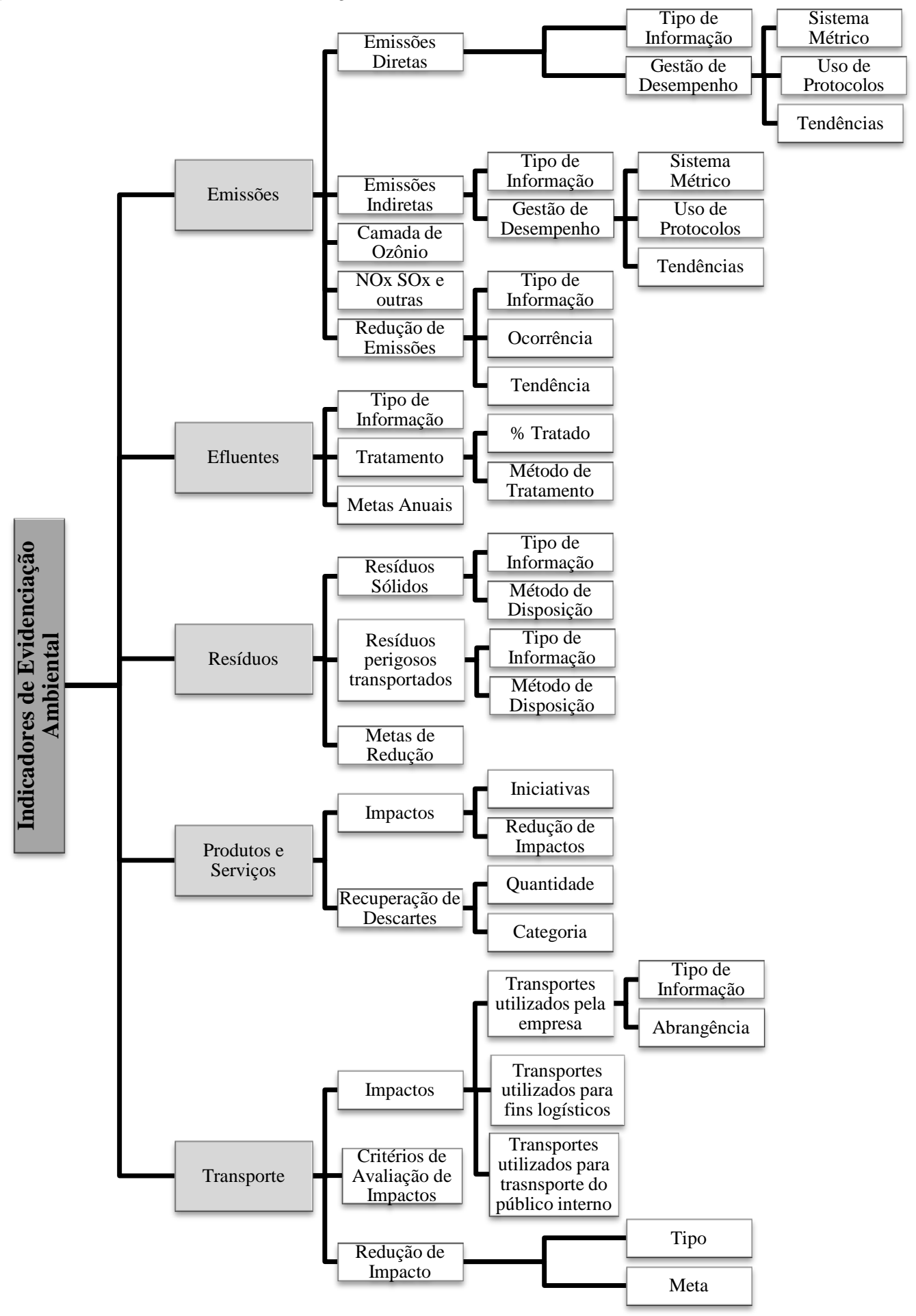

Fonte: Adaptado do GRI (2013).

Na planilha eletrônica do software excel, foram preenchidos ao lado de cada critério e subcritério, seu devido nível. A análise referente aos níveis para cada empresa da amostra foi realizada em cada ano analisado e referente a cada relatório (RS e RA) de forma separada.

Depois de verificados e preenchidos todos os níveis de evidenciação das empresas sobre os aspectos analisados tanto no Relatório Anual como no Relatório de Sustentabilidade os dados foram tabulados de acordo com o nível de N1 a N8 para 1 a 8. Na sequência, foram 
somados os níveis dos Relatórios de Sustentabilidade com os níveis dos Relatórios Anuais de cada empresa analisada para se obter o peso, o grau de evidenciação ambiental individual, de cada ano, a partir do método T-ODA. Caso a empresa não divulgasse os seus relatórios (RS e RA), considerou-se que esta tivesse um nível de divulgação 1 (N1) para todos os critérios e subcritérios, o que é considerado um nível comprometedor de evidenciação ambiental.

\subsubsection{Método T-ODA}

No método de análise multicritério Trade-Off Decision Analysis (T-ODA) inicialmente deve-se estabelecer a importância de cada critério para comparar cada critério com os demais (MEIRELES; SANCHES, 2009). Na construção e utilização de um modelo que estabelece prioridades, fundamentado no T-ODA, tem-se as seguintes etapas: a especificação do objetivo da decisão; a definição de critérios para tomar decisões; a definição da função objetivo; a comparação pivô; a comparação consistente dos critérios; a ponderação consistente dos critérios; o peso relativo dos fatores; e o cálculo da função objetivo e escolha.

A ponderação dos critérios é fundamental seja qual for o método utilizado. Na etapa comparação pivô o peso dos critérios é tratado como determinístico na avaliação das alternativas. Essa ponderação dos critérios é feita por meio de uma escala Trade Off e atende algumas etapas. Inicialmente é preciso fazer uma comparação de um critério, considerado pivô, com os demais critérios analisados. Para esses outros critérios é estabelecido um peso que seja comparativo com o critério pivô (MEIRELES; SANCHES, 2009). A atribuição dos valores para as avaliações das importâncias relativas de cada critério é apresentada por Meireles e Sanches (2009) da seguinte forma, como observado no Quadro 2.

Quadro 2 - Avaliação e valor dos critérios

\begin{tabular}{|c|c|}
\hline Avaliação & Valor \\
\hline Extremamente preferida & 3.000 \\
\hline Fortemente preferida & 2.000 \\
\hline Moderadamente preferida & 1.200 \\
\hline Igualmente preferida & 1.000 \\
\hline Moderadamente inferior & 0.833 \\
\hline Fortemente inferior & 0.500 \\
\hline Extremamente inferior & 0.333 \\
\hline
\end{tabular}

Fonte: Meireles e Sanches (2009).

Como neste estudo tem-se os aspectos de acordo com as diretrizes do GRI (2013), divididos em critérios e subcritérios, é necessário fazer essa avaliação e atribuir valores a todos estes critérios e subcritérios que serão analisados. No Quadro 2 é possível visualizar que parte-se do pivô (igualmente preferida) para estabelecer a comparação com os demais critérios de forma consistente. Conforme Meireles e Sanches (2009), este quadro deve ser entendido como uma orientação e está subordinado a valores que eventualmente foram medidos ou captados de alguma maneira.

O valor dos critérios não seguiu a proposta de Meireles e Sanches (2009). Estes foram obtidos por meio de uma adaptação em que se calculou o valor da informação para cada um dos aspectos analisados (emissões, efluentes, resíduos, produtos e serviços e transporte). Como aspecto pivô utilizou-se o valor da informação calculado para emissões e os demais pesos são relativos a este aspecto. Diante disso, a partir da Tabela 1 apresentam-se os aspectos e seus respectivos pesos utilizados no presente estudo em cada ano analisado.

Tabela 1 - Aspectos e pesos relativos

\begin{tabular}{|c|c|c|c|c|}
\hline \multirow{2}{*}{ Aspectos } & \multicolumn{4}{|c|}{ Pesos } \\
\hline & 2010 & 2011 & 2012 & 2013 \\
\hline Emissões* & 1,0000 & 1,0000 & 1,0000 & 1,0000 \\
\hline Efluentes & 0,8962 & 0,3692 & 0,4404 & 0,4597 \\
\hline Resíduos & 1,6406 & 1,1486 & 0,9575 & 1,3365 \\
\hline Produtos e Serviço & 1,3132 & 0,9647 & 1,1137 & 1,9097 \\
\hline Transporte & 0,0805 & 0,0615 & 0,0688 & 0,0598 \\
\hline (*) Critério pivô & & & & \\
\hline
\end{tabular}

Fonte: Meireles e Sanches (2009). 
Com o intuito de aclarar o método T-ODA, é apresentado um exemplo em que são tomadas oito empresas, que serão ranqueadas segundo dois grupos de perguntas. O primeiro aspecto é composto por três critérios/subcritérios e o segundo por dois critérios/subcritérios. Os dados são meramente ilustrativos, não possuindo qualquer relação com os valores coletados neste estudo. O modelo busca identificar 'quanto maior, melhor'.

\begin{tabular}{|c|c|c|c|c|c|}
\hline Empresa & Q1_A1 & Q2_A1 & Q3_A1 & Q1_A2 & Q2_A2 \\
\hline Empresa_1 & 4 & 2 & 0 & 8 & 4 \\
\hline Empresa_2 & 5 & 1 & 0 & 7 & 3 \\
\hline Empresa_3 & 6 & 3 & 0 & 3 & 6 \\
\hline Empresa_4 & 5 & 2 & 1 & 6 & 7 \\
\hline Empresa_5 & 2 & 3 & 1 & 2 & 6 \\
\hline Empresa_6 & 6 & 2 & 0 & 3 & 5 \\
\hline Empresa_7 & 5 & 1 & 1 & 1 & 4 \\
\hline Empresa_8 & 1 & 2 & 0 & 5 & 3 \\
\hline
\end{tabular}

Fonte: Dados da pesquisa.

Antes da inicialização do método T-ODA foi necessária uma preparação prévia dos dados para a verificação da importância de cada informação. Essa preparação seguiu a metodologia adota por Krylovas et al. (2014, p. 1124). A verificação do peso de cada informação no bloco (A1 e A2), foi obtida somando os pontos de cada coluna como pode ser visualizado no Quadro 4.

Quadro 4 - Exemplo: Passo 2

\begin{tabular}{|c|c|c|c|c|c|}
\hline Empresa & Q1_A1 & Q2_A1 & Q3_A1 & Q1_A2 & Q2_A2 \\
\hline Empresa_1 & 4 & 2 & 0 & 8 & 4 \\
\hline Empresa_2 & 5 & 1 & 0 & 7 & 3 \\
\hline Empresa_3 & 6 & 3 & 0 & 3 & 6 \\
\hline Empresa_4 & 5 & 2 & 1 & 6 & 7 \\
\hline Empresa_5 & 2 & 3 & 1 & 2 & 6 \\
\hline Empresa_6 & 6 & 2 & 0 & 3 & 5 \\
\hline Empresa_7 & 5 & 1 & 1 & 1 & 4 \\
\hline Empresa_8 & 1 & 2 & 0 & 5 & 3 \\
\hline Soma & 34 & 16 & 3 & 39 & 38 \\
\hline
\end{tabular}

Fonte: Dados da pesquisa.

Os somatórios de cada coluna foram acumulados para cada aspecto, totalizando: $\sum A 1=53$ e $\sum A 2=77$. Em seguida as somas de cada coluna foram dividas pela soma do aspecto, obtendo-se: $\quad w_{1,1}=0,642 ; w_{2,1}=0,302 ; w_{3,1}=0,056 ; w_{1,2}=0,506 ; w_{2,2}=$ 0,494 . A soma dos pesos de cada aspecto resulta em 1 .

A seguir os dados iniciais foram unificados em dois aspectos, por meio de uma média ponderada em que os valores $w_{i, j}$ (i é a questão, j é o aspecto) foram utilizados. Após foram calculadas as variâncias de cada aspecto e por meio desta, calculado o peso de cada um dos aspectos. O método T-ODA possui critérios de peso comparativo, conforme apresentado no Quadro 16, tendo como referência o pivô. Meireles e Sanches (2009, p. 42) sustentam que ela é "orientativa e subordina-se a valores eventualmente medidos e captados de alguma forma". Com o peso dos critérios é possível estabelecer a relação de Trade-Off (RTO) e o inverso da relação Trade-Off (iRTO) do método T-ODA.

Quadro 5 - Exemplo: Passo 3
\begin{tabular}{|c|c|c|}
\hline Empresa & A1 & A2 \\
\hline Empresa_1 & 3,169 & 6,026 \\
\hline Empresa_2 & 3,509 & 5,026 \\
\hline Empresa_3 & 4,755 & 4,481 \\
\hline Empresa_4 & 3,868 & 6,494 \\
\hline Empresa_5 & 2,245 & 3,974 \\
\hline Empresa_6 & 4,452 & 6,013 \\
\hline Empresa_7 & 3,566 & 2,241 \\
\hline Empresa_8 & 1,245 & 4,013 \\
\hline Variância & 1,316 & 1,811 \\
\hline
\end{tabular}


\begin{tabular}{|c|c|c|}
\hline Valor do Critério (\%) & 42,1 & 57,9 \\
\hline
\end{tabular} Fonte: Dados da pesquisa.

A seguir é apresentada a matriz de priorização correspondente (os valores do RTO e iRTO). Como há apenas dois aspectos estes são calculados como sendo: $\frac{0,421}{0,579}=0,727 \mathrm{e}$ $\frac{0,579}{0,421}=1,375$. Por meio destes obtém-se a comparação consistente dos aspectos conforme demonstrado no Quadro 6.

Quadro 6 - Exemplo: Passo 4

\begin{tabular}{|l|c|c|c|c|}
\hline RTO/iRTO & Aspecto-1 & Aspecto-2 & Soma & Peso \\
\hline Aspecto-1 & & 0,727 & 0,727 & 0,346 \\
\hline Aspecto-2 & 1,375 & & 1,375 & 0,654 \\
\hline Soma & 1,375 & 0,727 & 2,102 & 1 \\
\hline
\end{tabular}

Fonte: Dados da pesquisa.

Como o aspecto geral (quanto maior melhor) já foi estabelecido, o problema é reduzido em determinar o valor máximo da seguinte expressão:

$$
\text { Função Objetivo }=\operatorname{Max}\left\{\begin{array}{l}
E_{1}=0,346 A_{1,1}+0,654 A_{2,1} \\
E_{2}=0,346 A_{1,2}+0,654 A_{2,2} \\
E_{3}=0,346 A_{1,3}+0,654 A_{2,3} \\
E_{4}=0,346 A_{1,4}+0,654 A_{2,4} \\
E_{5}=0,346 A_{1,5}+0,654 A_{2,5} \\
E_{6}=0,346 A_{1,6}+0,654 A_{2,6} \\
E_{7}=0,346 A_{1,7}+0,654 A_{2,7} \\
E_{8}=0,346 A_{1,8}+0,654 A_{2,8}
\end{array}\right.
$$

Para resolver o sistema é necessário introduzir os aspectos com seus pesos normalizados. O exemplo tomou como pivô (valor de referência) a Empresa-1, a análise do primeiro aspecto. O resultado da análise da relação Trade-Off do primeiro aspecto ficou estabelecido de acordo com o que consta no Quadro 7.

Quadro 7 - Exemplo: Passo 5
\begin{tabular}{|c|c|c|}
\hline Empresa & Aspecto & Índice \\
\hline E-1 & 3,169 & 1 \\
\hline E-2 & 3,509 & 1,107 \\
\hline E-3 & 4,755 & 1,500 \\
\hline E-4 & 3,868 & 1,220 \\
\hline E-5 & 2,245 & 0,708 \\
\hline E-6 & 4,452 & 1,405 \\
\hline E-7 & 3,566 & 1,125 \\
\hline E-8 & 1,245 & 0,393 \\
\hline
\end{tabular}

Fonte: Dados da pesquisa.

A seguir, por meio do Quadro 8 é apresentada a matriz com os valores RTO e iRTO.

Quadro 8 - Exemplo: Passo 6
\begin{tabular}{|l|l|l|l|l|l|l|l|l|l|l|}
\hline RTO & E-1 & E-2 & E-3 & E-4 & E-5 & E-6 & E-7 & E-8 & Soma & $\%$ \\
\hline E-1 & & 0,903 & 0,667 & 0,820 & 1,412 & 0,712 & 0,889 & 2,544 & 7,947 & 11,7 \\
\hline E-2 & 1,107 & & 0,738 & 0,907 & 1,564 & 0,788 & 0,984 & 2,817 & 8,905 & 13,2 \\
\hline E-3 & 1,500 & 1,355 & & 1,230 & 2,119 & 1,067 & 1,333 & 3,817 & 12,421 & 18,4 \\
\hline E-4 & 1,220 & 1,102 & 0,813 & & 1,723 & 0,868 & 1,084 & 3,104 & 9,914 & 14,6 \\
\hline E-5 & 0,708 & 0,640 & 0,472 & 0,580 & & 0,504 & 0,629 & 1,801 & 5,334 & 7,9 \\
\hline E-6 & 1,405 & 1,269 & 0,937 & 1,152 & 1,984 & & 1,249 & 3,575 & 11,571 & 17,1 \\
\hline E-7 & 1,125 & 1,016 & 0,750 & 0,922 & 1,589 & 0,801 & & 2,863 & 9,066 & 13,4 \\
\hline E-8 & 0,393 & 0,355 & 0,262 & 0,322 & 0,555 & 0,280 & 0,349 & & 2,516 & 3,7 \\
\hline
\end{tabular}

Fonte: Dados da pesquisa.

O mesmo acontece com o segundo aspecto (A2), em que as empresas são comparadas entre si. Também a Empresa-1 é utilizada como pivô. 
Quadro 9 - Exemplo: Passo 7

\begin{tabular}{|c|c|c|}
\hline & Fator & \multicolumn{1}{|c|}{ Índice } \\
\hline Empresa & 6,026 & 0,834 \\
\hline E-1 & 5,026 & 0,744 \\
\hline E-2 & 4,481 & 1,077 \\
\hline E-3 & 6,494 & 0,659 \\
\hline E-5 & 3,974 & 0,998 \\
\hline E-6 & 6,013 & 0,372 \\
\hline E-7 & 2,241 & 0,666 \\
\hline
\end{tabular}

Fonte: Dados da pesquisa.

De similar modo é apresentada a matriz com os valores RTO e iRTO do segundo aspecto, de acordo com o Quadro 10.

Quadro 10 - Exemplo: Passo 8
\begin{tabular}{|c|c|c|c|c|c|c|c|c|c|c|}
\hline RTO & E-1 & E-2 & E-3 & E-4 & E-5 & E-6 & E-7 & E-8 & Soma & $\%$ \\
\hline E-1 & & 1,199 & 1,344 & 0,929 & 1,517 & 1,002 & 2,688 & 1,502 & 10,181 & 16,2 \\
\hline E-2 & 0,834 & & 1,121 & 0,774 & 1,266 & 0,836 & 2,242 & 1,252 & 8,325 & 13,2 \\
\hline E-3 & 0,744 & 0,892 & & 0,691 & 1,129 & 0,745 & 2,000 & 1,117 & 7,318 & 11,6 \\
\hline E-4 & 1,077 & 1,291 & 1,448 & & 1,634 & 1,079 & 2,895 & 1,617 & 11,041 & 17,5 \\
\hline E-5 & 0,659 & 0,790 & 0,886 & 0,612 & & 0,660 & 1,772 & 0,854 & 6,233 & 9,9 \\
\hline E-6 & 0,998 & 1,197 & 1,341 & 0,927 & 1,514 & & 2,683 & 1,498 & 10,158 & 16,1 \\
\hline E-7 & 0,372 & 0,446 & 0,500 & 0,345 & 0,564 & 0,373 & & 0,559 & 3,159 & 5,0 \\
\hline E-8 & 0,666 & 0,799 & 0,896 & 0,618 & 1,170 & 0,667 & 1,790 & & 6,606 & 10,5 \\
\hline
\end{tabular}

Fonte: Dados da pesquisa.

A pontuação de cada empresa fica assim estabelecida.

$$
\text { Pontuação }=\left\{\begin{array}{c}
E_{1}=0,346 \times 11,7+0,654 \times 16,2=14,643 \\
E_{2}=0,346 \times 13,2+0,654 \times 13,2=13,2 \\
E_{3}=0,346 \times 18,4+0,654 \times 11,6=13,953 \\
E_{4}=0,346 \times 14,6+0,654 \times 17,5=16,497 \\
E_{5}=0,346 \times 7,9+0,654 \times 9,9=9,208 \\
E_{6}=0,346 \times 17,1+0,654 \times 16,1=16,446 \\
E_{7}=0,346 \times 13,4+0,654 \times 5,0=7,906 \\
E_{8}=0,346 \times 3,7+0,654 \times 10,5=8,147
\end{array}\right.
$$

A leitura do ranking é feita em ordem decrescente, assim a ordenação formada segue a sequência: Empresa-4 (16,497 pontos), Empresa-6 (16,446 pontos), Empresa-1 (14,643 pontos), Empresa-2 (13,2 pontos), Empresa-3 (13,953 pontos), Empresa-5 (9,208 pontos), Empresa-8 (8,147 pontos) e Empresa-7 (7,906 pontos).

Este mesmo conjunto de procedimentos foi utilizado com as 97 empresas analisadas e com os cinco aspectos que apresentam diversos critérios e subcritérios.

\section{DESCRIÇÃO E ANÁLISE DOS DADOS}

De posse dos rankings anuais, foi estabelecido um ranking geral em relação ao grau de evidenciação das empresas analisadas e o período. Para constituir esse ranking geral a partir dos rankings parciais (anuais), verificou-se a evolução das organizações durante os quatro anos analisados por meio de um sistema de pontos corridos.

Na Tabela 2 apresenta-se o score obtido a partir do método T-ODA e o ranking das empresas analisadas no que diz respeito à evidenciação ambiental nos quatro anos analisados e sua posição final.

Tabela 2 - Grau de evidenciação e ranking de evidenciação ambiental

\begin{tabular}{|c|c|c|c|c|c|c|c|c|c|}
\hline \multirow[t]{2}{*}{ Empresas } & \multicolumn{2}{|c|}{2010} & \multicolumn{2}{|c|}{2011} & \multicolumn{2}{|c|}{2012} & \multicolumn{2}{|c|}{2013} & \multirow{2}{*}{$\begin{array}{c}\begin{array}{c}\text { Ranking } \\
\text { final }\end{array} \\
\text { Posição }\end{array}$} \\
\hline & Score & Posição & Score & Posição & Score & Posição & Score & Posição & \\
\hline$\overline{\text { Duratex }}$ & 4,0110 & 2 & 3,6753 & 1 & 2,8970 & 7 & 3,4020 & 5 & 1 \\
\hline Ecorodovias & 3,6690 & 3 & 3,0220 & 5 & 3,1233 & 4 & 3,4130 & 4 & 2 \\
\hline Energias BR & 3,6463 & 4 & 3,4816 & 3 & 2,7618 & 8 & 3,0298 & 9 & 3 \\
\hline P. Açúcar & 2,8840 & 7 & 3,0602 & 4 & 2,5780 & 10 & 3,2265 & 7 & 4 \\
\hline Copel & 3,3301 & 5 & 3,5445 & 2 & 2,5144 & 11 & 2,6049 & 11 & 5 \\
\hline
\end{tabular}




\begin{tabular}{|c|c|c|c|c|c|c|c|c|c|}
\hline Cemig & 2,1130 & 16 & 2,0971 & 18 & 2,9250 & 6 & 3,4886 & 2 & 6 \\
\hline BM\&FBovespa & 2,8048 & 9 & 2,6021 & 12 & 2,4131 & 12 & 2,7756 & 10 & 7 \\
\hline Natura & 2,1617 & 15 & 2,8144 & 9 & 2,9553 & 5 & 2,3243 & 14 & 7 \\
\hline Petrobrás & 2,7951 & 10 & 2,7532 & 10 & 2,1337 & 20 & 2,1783 & 16 & 9 \\
\hline$\overline{\text { AES Tietê }}$ & 2,8054 & 8 & 2,5774 & 13 & 2,1483 & 19 & 2,1717 & 17 & 10 \\
\hline Braskem & 1,9786 & 20 & 1,7136 & 29 & 3,2221 & 2 & 3,3818 & 6 & 10 \\
\hline Eletrobrás & 1,2503 & 41 & 2,4140 & 15 & 3,1655 & 3 & 3,8811 & 1 & 12 \\
\hline BRF - Brasil Foods & 3,2604 & 6 & 3,0056 & 6 & 3,3789 & 1 & 0 & 48 & 13 \\
\hline Even & 1,8702 & 24 & 2,6339 & 11 & 2,2560 & 14 & 2,3051 & 15 & 14 \\
\hline Copasa & 2,3085 & 14 & 2,9639 & 7 & 2,0736 & 21 & 1,1165 & 42 & 15 \\
\hline Eletropaulo & 2,4661 & 11 & 1,7516 & 27 & 1,8767 & 29 & 2,1280 & 20 & 16 \\
\hline$\overline{\mathrm{JBS}}$ & 1,2542 & 40 & 1,4042 & 39 & 2,6958 & 9 & 3,4760 & 3 & 17 \\
\hline Klabin & 2,3863 & 12 & 2,5596 & 14 & 1,8714 & 30 & 1,6727 & 36 & 18 \\
\hline Banco Bradesco & 2,0454 & 17 & 2,0913 & 19 & 1,7117 & 36 & 2,0140 & 23 & 19 \\
\hline Vale & 1,5793 & 30 & 1,5092 & 34 & 2,1522 & 17 & 2,1445 & 19 & 20 \\
\hline CPFL Energia & 1,9108 & 22 & 1,9214 & 22 & 1,7116 & 37 & 2,0655 & 21 & 21 \\
\hline CCR & 1,9908 & 19 & 1,4067 & 38 & 2,1513 & 18 & 1,8427 & 31 & 22 \\
\hline Embraer & 0 & 52 & 2,2896 & 16 & 2,0563 & 22 & 2,1693 & 18 & 23 \\
\hline Souza cruz & 1,6338 & 29 & 0 & 52 & 2,2028 & 16 & 2,5975 & 12 & 24 \\
\hline Tractebel & 2,3342 & 13 & 2,1899 & 17 & 1,7835 & 33 & 0,8718 & 47 & 25 \\
\hline Ambev & 4,8399 & 1 & 2,9477 & 8 & 0 & 55 & 0 & 48 & 26 \\
\hline Lojas Renner & 1,2806 & 37 & 1,0748 & 46 & 1,9484 & 26 & 3,1769 & 8 & 27 \\
\hline Suzano Papel & 1,9430 & 21 & 1,7894 & 24 & 2,0188 & 24 & 0 & 48 & 27 \\
\hline Weg & 1,1986 & 43 & 2,0636 & 20 & 1,9595 & 25 & 1,7577 & 33 & 29 \\
\hline Fibria & 1,8705 & 23 & 1,7055 & 30 & 1,5422 & 40 & 1,8175 & 32 & 30 \\
\hline Sabesp & 1,7421 & 26 & 1,7419 & 28 & 1,3302 & 45 & 1,9630 & 27 & 31 \\
\hline Tim Participações & 1,4720 & 31 & 1,4546 & 37 & 2,3818 & 13 & 0 & 48 & 32 \\
\hline Sul América & 2,0198 & 18 & 1,8843 & 23 & 1,5138 & 41 & 0 & 48 & 33 \\
\hline Banco do Brasil & 1,6382 & 28 & 0 & 52 & 1,8013 & 32 & 2,0054 & 25 & 34 \\
\hline Marfrig & 1,1554 & 48 & 1,0586 & 49 & 2,0257 & 23 & 2,0372 & 22 & 35 \\
\hline Porto Seguro & 1,4153 & 33 & 0 & 52 & 1,2457 & 46 & 2,4484 & 13 & 36 \\
\hline Light & 1,3814 & 35 & 0 & 52 & 1,8460 & 31 & 1,9431 & 28 & 37 \\
\hline Marcopolo & 0 & 52 & 1,7801 & 26 & 1,4614 & 42 & 1,9337 & 29 & 38 \\
\hline Itaú Unibanco & 1,4124 & 39 & 1,3382 & 44 & 1,2371 & 47 & 2,0134 & 24 & 39 \\
\hline Santander & 0 & 52 & 0 & 52 & 2,2444 & 15 & 1,7287 & 35 & 39 \\
\hline Ultrapar & 1,4598 & 32 & 1,5448 & 32 & 1,3699 & 44 & 0 & 48 & 41 \\
\hline Cosan & 0 & 52 & 0 & 52 & 1,8947 & 27 & 1,9844 & 26 & 42 \\
\hline Banrisul & 0 & 52 & 1,7849 & 25 & 1,4302 & 43 & 1,6183 & 38 & 43 \\
\hline Multiplant & 1,2767 & 38 & 2,0353 & 21 & 0,9599 & 52 & 0 & 48 & 44 \\
\hline Randon Part. & 0 & 52 & 1,6880 & 31 & 1,8936 & 28 & 0 & 48 & 44 \\
\hline Gol & 1,8121 & 25 & 1,5283 & 33 & 0 & 55 & 0 & 48 & 46 \\
\hline Oi & 0 & 52 & 0 & 52 & 1,7127 & 35 & 1,8494 & 30 & 47 \\
\hline Itausa & 1,2666 & 34 & 1,1124 & 41 & 0 & 55 & 1,0730 & 44 & 48 \\
\hline Hypermarcas & 1,1554 & 47 & 1,0798 & 45 & 1,0937 & 51 & 1,7488 & 34 & 49 \\
\hline Valetron & 1,3698 & 36 & 1,3863 & 40 & 0 & 55 & 0 & 48 & 50 \\
\hline Rossi Resid. & 1,6881 & 27 & 0 & 52 & 0 & 55 & 0 & 48 & 51 \\
\hline Telefônica Brasil & 1,1379 & 49 & 1,0507 & 50 & 1,6335 & 38 & 0,9525 & 45 & 51 \\
\hline Estácio Part. & 0 & 52 & 0 & 52 & 1,5712 & 39 & 1,3026 & 40 & 53 \\
\hline$\overline{\mathrm{MRV}}$ & 1,1677 & 45 & 1,2082 & 42 & 1,1214 & 50 & 0 & 48 & 54 \\
\hline Cielo & 0 & 52 & 0 & 52 & 1,7824 & 34 & 0 & 48 & 55 \\
\hline Cyrela Realty & 1,1554 & 46 & 1,0586 & 48 & 0,8693 & 53 & 1,4921 & 39 & 55 \\
\hline Lojas Americanas & 1,1677 & 44 & 1,0745 & 47 & 1,1581 & 49 & 0 & 48 & 57 \\
\hline Sid nacional & 1,2299 & 42 & 1,1132 & 43 & 0 & 55 & 0 & 48 & 57 \\
\hline Valid & 0 & 52 & 1,4945 & 35 & 0 & 55 & 0 & 48 & 59 \\
\hline Minerva & 0 & 52 & 1,4888 & 36 & 0 & 55 & 0 & 48 & 60 \\
\hline Gerdau & 0 & 52 & 0 & 52 & 0 & 55 & 1,6198 & 37 & 61 \\
\hline Dasa & 0 & 52 & 0 & 52 & 0,8693 & 54 & 1,2724 & 41 & 62 \\
\hline Magazine Luiza & 0 & 52 & 0 & 52 & 1,2215 & 48 & 0 & 48 & 63 \\
\hline Taesa & 0 & 52 & 0 & 52 & 0 & 55 & 1,0940 & 43 & 64 \\
\hline ALL Amer. Lat. Log. & 1,1225 & 51 & 1,0305 & 51 & 0 & 55 & 0 & 48 & 65 \\
\hline Arteris & 0 & 52 & 0 & 52 & 0 & 55 & 0,8865 & 46 & 65 \\
\hline Gafisa & 1,1319 & 50 & 0 & 52 & 0 & 55 & 0 & 48 & 65 \\
\hline
\end{tabular}

Fonte: Dados da pesquisa.

Inicialmente destaca-se que 67 empresas das 97 apresentaram pelo menos uma informação ambiental em seus Relatórios Anuais e de Sustentabilidade. As demais, ou seja, 30 empresas obtiveram score igual a zero em todos os anos analisados, ficando com a última posição no ranking de evidenciação ambiental e, estas não foram apresentadas no ranking. 
Isso demonstra que um grande número de empresas não está divulgando informações suficientes sobre os aspectos ambientais em seus relatórios.

Conforme a Tabela 2, observa-se que em relação ao resultado do método T-ODA (score) obteve-se o grau de evidenciação e destacam-se as empresas que lideraram o ranking em cada ano: Ambev (2010), Duratex (2011), BRF Brasil Foods (2012) e a Eletrobrás (2013). No ano de 2010 verificou-se o maior grau, se comparado aos demais períodos. Além disso, neste mesmo ano e em 2011, 51 empresas divulgaram informações ambientais em seus relatórios. Em 2012 foram 54 empresas que apresentaram alguma informação ambiental e no último ano, (2013), apenas 47.

Salienta-se que as empresas Duratex, Ecorodovias, Energias BR e Pão de Açúcar ficaram classificadas nas posições iniciais no ranking em todos os anos analisados, no que diz respeito ao grau de evidenciação ambiental. Estas empresas são as que mais evidenciaram informações sobre as emissões, efluentes, resíduos, produtos e serviços e transportes neste período. Das vinte empresas melhor posicionadas no ranking de cada ano, foi possível constatar que a maioria destas apenas mudava a sua colocação de um ano para o outro.

\section{CONSIDERAÇÕES FINAIS}

Conclui-se que as empresas podem aumentar o grau de evidenciação ambiental se divulgarem mais informações em seus relatórios sobre suas ações no meio ambiente, principalmente sobre o aspecto transporte, visto que poucas das empresas analisadas apresentam essa informação em seus relatórios. Dessa forma, passarão a transmitir uma imagem ainda melhor à sociedade.

Destaca-se que as empresas não possuem um padrão de divulgação nos seus relatórios sobre as informações ambientais, visto que estas possuem comportamentos diferentes umas das outras e, divulgam diferentes informações de um ano para o outro, o que indica seleção de informações para a divulgação ambiental. Entende-se que o nível de divulgação é insuficiente e algumas das causas são a falta de divulgação das práticas ambientais, a ineficiência de controles e a falta de motivação para divulgação, o que representa uma lacuna de pesquisa.

Mesmo que tais relacionamentos foram identificados neste estudo, verificou-se que é importante as empresas divulgarem um maior número de informações para serem legitimadas na sociedade. Isso acontece quando as empresas apresentam que suas atividades em prol do meio ambiente estão de acordo com os valores e regras que são estabelecidas pela sociedade. Ressalta-se que está cada vez mais difícil as organizações atingirem a legitimidade. Dessa forma, se as empresas evidenciarem o máximo de informações sobre as suas ações ambientais nos seus relatórios, estas poderão alcançar a legitimidade desejada de forma rápida.

\section{REFERÊNCIAS BIBLIOGRÁFICAS}

[1] ALBERTINI, Elisabeth. A descriptive analysis of environmental disclosure: A longitudinal study of French companies. Journal of Business Ethics, v. 121, n. 2, p. 233 254, 2013.

[2] COSTA, Rodrigo Simão da. Evidenciação Contábil das Informações Ambientais: Uma Análise das Empresas do Setor de Papel e Celulose da BOVESPA. 2006. 146 f. Dissertação (Mestrado em Ciências Contábeis e Atuárias) - Pontifícia Universidade Católica de São Paulo: PUC-SP, Programa de Estudos Pós-Graduados em Ciências Contábeis e Financeiras, São Paulo, 2006.

[3] GARCÍA-SÁNCHEZ, Isabel-María; FRÍAS-ACEITUNO, José-Valeriano; RODRÍGUEZ-DOMÍNGUEZ, Luis. Determinants of corporate social disclosure in Spanish local governments. Journal of Cleaner Production, v. 39, p. 60-72, 2013.

[4] GRI. Global Reporting Initiative. Elaboração de relatórios de sustentabilidade. 2013. Disponível

<https://www.globalreporting.org/languages/Portuguesebrazil/Pages/Elaboração-derelatórios-de-sustentabilidade.aspx>. Acesso em: 13 Set. 2014. 
[5] KRESPI, Nayane Thais; UTZIG, Mara Jaqueline Santore; DALLABONA, Lara Fabiana; SCARPIN, Jorge Eduardo. Disclosure of information environmental analysis under the social aspect of companies listed on ISE. Custos e @ gronegócio on line, v. 8, n. 4, p. 147-170, 2012.

[6] KRYLOVAS, Aleksandras; ZAVADSKAS, Edmundas Kazimieras; KOSAREVA, Natalja; DADELO, Stanislav. New KEMIRA Method for Determining Criteria Priority and Weights in Solving MCDM Problem. International Journal of Information Technology \& Decision Making, v. 13, n. 06, p. 1119-1133, 2014.

[7] MEIRELES, Manuel; SANCHES, Cida. ST-ODA: Strategic Trade-Off Decision Analysis - Processo de tomada de decisões gerenciais multicritério subordinadas à vantagem competitiva. 1. Ed. São Paulo: FACCAMP, 2009.

[8] MENG, X. H.; ZENG, S. X.; TAM, C. M.; XU, X. D. Whether top executives' turnover influences environmental responsibility: From the perspective of environmental information disclosure. Journal of business ethics, v. 114, n. 2, p. 341-353, 2013.

[9] NOSSA, Valcemiro. Disclosure ambiental: uma análise do conteúdo dos relatórios ambientais de empresas do setor de papel e celulose em nível internacional. São Paulo, 2002. 249 f. Tese (Doutorado em Ciências Contabéis) - Programa de Pós-Graduação em Ciências Contabéis, Departamento de Contabilidade e Atuária, Faculdade de Economia, Administração e Contabilidade da Universidade de São Paulo, São Paulo, 2002.

[10] OLIVEIRA, Araceli Farias de; MACHADO, Débora Gomes; BEUREN, Ilse Maria. Disclosure Ambiental de Empresas de Setores Potencialmente Poluidores Listadas no Índice de Sustentabilidade Empresarial (ISE). Revista de Gestão Social e Ambiental, v. 6, n. 1, p. 20-37 2012.

[11] ROSA, Fabricia Silva da; GUESSER, Tatiana; HEIN, Nelson; PFITSCHER, Elisete Dahmer; LUNKES, Rogério João Lunkes. Environmental impact management of Brazilian companies: analyzing factors that influence disclosure of waste, emissions, effluents, and other impacts. Journal of Cleaner Production, p. 1-13, 2013.

[12] ROSA, Fabricia Silva da; LUNKES, Rogério João; HEIN, Nelson; VOGT, Mara, DEGENHART, Larissa. Analysis of the determinants of disclosure of environmental impacts of Brazilian companies. Global Advanced Research Journals, vol. 3, n. 6, p. 249-266, 2014.

[13] ROSSONI, Cláudio Farias. Decisão Multicritério - Uma pesquisa experimental para avaliação da percepção dos gestores de MPE acerca do modelo de tomada de decisão multicritério T-ODA quanto à sua aplicabilidade. 2011 . 241 f. Dissertação (Mestrado em Administração)-Faculdade Campo Limpo Paulista - FACCAMP, Programa de Mestrado em Administração, Campo Limpo Paulista, 2011.

[14] VILLIERS, Charl de; LOW, Mary; SAMKIN, Grant. The institutionalisation of mining company sustainability disclosures. Journal of Cleaner Production, p. 1-8, 2014.

[15] WANG, Hua; BERNELL, David. Environmental Disclosure in China: An Examination of the Green Securities Policy. The Journal of Environment \& Development, v. 22, n. 4, p. 339-369, 2013. 